\title{
Audit of a tuberculosis contact tracing clinic
}

\author{
S F Hussain, R Watura, Beryl Cashman, I A Campbell, M R Evans
}

Abstract

Objective-To determine the efficiency of tuberculosis contact tracing in South Glamorgan 1987-9.

Design-Review of records of contact tracing clinic and of data from the Mycobacterium Reference Unit. The clinic's practice was compared with 1983 British Thoracic Society's recommendations.

Setting-Health authority tuberculosis control programme.

Main outcome measures-Proportion of contacts screened, follow up attendance rates, number of secondary cases detected, and quality of record keeping.

Results -101 index patients and 611 contacts were identified. $596(97.5 \%)$ contacts were screened, of whom 139 should not have been. Of 356 contacts requiring a Heaf test, 237 were tested, seven refused the test, and 112 had chest radiography without a Heaf test. 95 contacts were unnecessarily tested. 87 contacts had chest radiography unnecessarily and seven should have had radiography but did not. 34 contacts were given follow up appointments inappropriately and seven were overlooked for follow up. Tuberculosis was diagnosed in five asymptomatic contacts, all at initial screening and all close contacts of index patients with pulmonary disease.

Conclusion-Inadequacy of data, non-adherence to contact tracing guidelines, and failure to define the term highly infectious index case resulted in many contacts being unnecessarily screened or followed up.

Implications - The efficiency of tracing contacts would be improved by specifying smear results and ethnic origin of the index case on the notification form, clearly classifying contacts as close or casual, and clearly defining the term highly infectious.

\section{Introduction}

Contact tracing is widely used to detect tuberculosis among asymptomatic contacts. In South Glamorgan about 40 new cases of tuberculosis are notified each year (population 400000 , incidence $1 / 10000 /$ year). In 1978 the risk of close contacts of patients with smear positive disease developing active tuberculosis was considered to be $9 \%$ for Asians and $12 \%$ for nonAsians, and for contacts of those with smear negative disease the risk was $2.8 \%$ and $0.5 \%$ respectively.' Casual contacts have a low risk of tuberculosis $(0.3 \%)$ and close contacts of index cases with non-pulmonary tuberculosis have a risk of $1 \% .^{1}$ The British Thoracic Society's 1990 code of practice ${ }^{2}$ and recent studies ${ }^{3-5}$ suggest that these 1978 figures are an overestimate of today's risk. We audited our district's tuberculosis contact tracing clinic using the British Thoracic Society's 1983 guidelines $^{6}$ as the standard against which to compare our practice and suggest changes to be implemented.

\section{Subjects and methods}

Data on all patients with tuberculosis in South Glamorgan between May 1987 and December 1989 were extracted from the contact tracing clinic, and further laboratory data were obtained from the Public
Health Laboratory Service Mycobacterium Reference Unit, Cardiff. For each index patient we determined age, sex, ethnic origin (Indian subcontinent or other), tuberculosis site, and results of smear test and culture. Data recorded on contacts included age, sex, ethnic origin, BCG vaccination, and follow up details. Contacts were classified as close or casual. A close contact was defined as a member of the same household as the notified case who shared kitchen or bathroom facilities, or both, or a close associate such as a relative who visited frequently, a girlfriend, or a boyfriend. All other contacts were regarded as casual.

The contact tracing procedure, based on the British Thoracic Society's 1983 recommendations, ${ }^{6}$ was as follows: contacts were screened with the Heaf test or chest radiography, or both. Contacts aged above 50 years were not given the Heaf test and those under 16 years did not have radiography unless the Heaf test result was positive. Close contacts who were Heaf grade $0-1$ and who had not received BCG vaccination were vaccinated and discharged. Those with Heaf grade 2-4 or abnormal chest radiographs, or both, were followed up in the contact tracing clinic or referred to the chest clinic. For index patients not from the Indian subcontinent with smear positive pulmonary disease and all index patients from the Indian subcontinent with pulmonary disease, close contacts were followed up with chest radiography at six monthly intervals for 12 and 24 months respectively. Casual contacts were screened only if the index patient proved to be highly infectious. However, the term highly infectious was not defined and all patients with smear positive disease were considered to be highly infectious by the contact team.

Results were analysed with the survey plus computer package.

Results

INITIAL SCREENING

We identified 101 index patients and 611 contacts170 close and 441 casual. Table I summarises the details of initial screening.

Of the 15 close contacts not initially screened, four were not contactable, two failed to attend clinic, one was already in hospital, and one was a neonate who was

TABLE I - Details of initial screening of contacts

\begin{tabular}{|c|c|}
\hline & No of contacts \\
\hline No identified & 611 \\
\hline No screened & 596 \\
\hline No unnecessarily screened & 139 \\
\hline \multicolumn{2}{|l|}{ Heaf test: } \\
\hline No tested & 332 \\
\hline Appropriately & 237 \\
\hline Unnecessarily & $95 *+$ \\
\hline No inappropriately omitted & 112 \\
\hline \multicolumn{2}{|l|}{ Chest radiography: } \\
\hline No having radiography & 376 \\
\hline Appropriately & \\
\hline Unnecessarily & 87 * \\
\hline No inappropriately omitted & 7 \\
\hline No referred to chest clinic: & 11 \\
\hline $\begin{array}{l}\text { Tuberculosis diagnosed } \\
\text { Tula }\end{array}$ & 5 \\
\hline Chemoprophylaxis & 4 \\
\hline Non-tuberculous radiographic changes & 2 \\
\hline
\end{tabular}

${ }^{*}$ Casual contacts of index patients with smear negative or non-pulmonary disease. tAge above 50 years.

Dr Campbell.

$B M \mathcal{1}$ 1992;304:1213-5 
given BCG vaccination without full screening. No reason for failing to screen the remaining seven could be ascertained.

Of the 356 contacts who required a Heaf test, seven refused, 237 were tested, and the remaining 112 had a chest radiograph without a Heaf test. A further 95 contacts had a Heaf test unnecessarily.

Heaf grade 0 was recorded in 179 contacts: 119 received $B C G$ vaccination and 14 refused. The remaining 46 were not offered vaccination ( 27 had received BCG vaccine previously and 19 were elderly contacts). Nineteen contacts were Heaf grade 1 , none of whom had had BCG vaccinations; none of the 19 was offered vaccination.

Of the 91 contacts with Heaf grades 2-4 (59 with grade 2, 17 grade 3,15 grade 4 ), seven had incomplete initial screening (no radiography), seven were discharged inappropriately (close contacts of index patients with pulmonary tuberculosis), 25 were followed up by the contact clinic, eight were referred to the chest clinic, and the remaining 44 were appropriately discharged.

Chest radiography was done in 376 contacts (112 close and 264 casual). Forty five close contacts (all but one below 16 years of age) did not have chest radiography, seven of whom were Heaf grade 2 . All close contacts over 50 years of age had chest radiography.

Eleven contacts were referred to the chest clinic because of abnormal appearances on chest radiography or Heaf grades 2-4. Tuberculosis was diagnosed in five contacts (two from the Indian subcontinent and three of other origin); in all cases the diagnosis was made on initial screening. One additional contact (from the Indian subcontinent and already in hospital) also had tuberculosis diagnosed but not through contact screening. Four contacts with Heaf grade 2-4 and aged less than 16 years were not referred to the chest clinic and did not receive chemoprophylaxis but were radiologically followed up in the contact clinic at six monthly intervals for one to two years.

All contacts who had tuberculosis diagnosed were below 26 years old and four were under 10 . All were close contacts and none was known to have been vaccinated. The index patients for five of these contacts had smear positive disease, and for one the index patient had smear negative disease but culture was positive. No contact of an index patient with culture negative pulmonary tuberculosis or non-pulmonary tuberculosis developed disease. Four contacts who received chemoprophylaxis were also close contacts of patients with smear positive pulmonary tuberculosis and had not been vaccinated.

The characteristics of the 108 contacts who were discharged after initial screening were reviewed. Seven with Heaf grades 2-4 were discharged inappropriately without a follow up appointment. The remainder were appropriately discharged.

\section{FOLLOW UP}

Follow up appointments were given to 70 contacts (table II). In 34 the appointment was not necessary: 23 were casual contacts, two were contacts of index patients with non-pulmonary tuberculosis, and nine were close contacts of index patients who had smear negative disease and were not from the Indian subcontinent. No new case of tuberculosis was diagnosed on follow up and none has been notified in South Glamorgan among the contacts who were inadequately screened or followed up.

\section{ADEQUACY OF DATA}

Data presented to the contact team regarding index patients were often inadequate. In three cases the site of tuberculosis was not mentioned and the smear test result was not recorded in 46 of the 71 patients with pulmonary disease. There was no formal system of liaison between the contact team and the microbiology services to establish the smear test result of index patients.

Data recorded by the contact team were also inadequate in some respects: ethnic group was not recorded for 91 index patients. In most cases no distinction was made between close and casual contacts (apart from home address). This meant that 132 casual contacts were inappropriately screened as if they were close contacts.

The reasons for not doing a Heaf test were not recorded for $18 \%$ of the contacts who were not tested. For those who were tested the results and subsequent BCG vaccination were fully documented. For nine of the 34 contacts inappropriately followed up the reason was inadequacy of data (smear test result of index patient unknown).

\section{Discussion}

The British Thoracic Society guidelines are widely used to formulate protocols for tuberculosis contact tracing. We have shown that they can serve as a useful standard against which to assess local performance. Published studies of tuberculosis contact tracing have not always addressed the structure, process, and outcome of the service adequately. In a recent paper on contact tracing, issues such as adherence to the tracing protocol, the proportion of contacts unnecessarily or inadequately screened, and the consequences of data 0 inadequacy were not addressed. ${ }^{5}$ We assessed the completeness of clinic records, the level of adherence to the protocol, patient attendance rates, and the effectiveness of the screening procedure.

Inadequacy of data and non-adherence to the protocol resulted in $23 \%$ of contacts being unnecessarily screened and $49 \%$ being unnecessarily followed up. Seven contacts were inadequately screened and a further seven were overlooked for follow up.

The site of tuberculosis, smear test results, and ethnic origin of theindex patient form the basis of contact tracing. However, the notification form at the time of study had no provision for ethnic origin and smear test results to be recorded. As a result in $65 \%$ of cases the contact team did not know the result of the smear test. In three cases even the site of tuberculosis was not reported on the notification form. Introduction of a formal system of communication between the contact team and the microbiology services could ensure that the smear test result is known.

TABLE II - Details of 70 contacts followed up by contact clinic

\begin{tabular}{|c|c|c|}
\hline \multirow[b]{2}{*}{$\begin{array}{l}\text { Follow up interval } \\
\text { and type of contact }\end{array}$} & \multicolumn{2}{|c|}{$\begin{array}{l}\text { No of contacts given appointment } \\
\text { (No attended) }\end{array}$} \\
\hline & $\begin{array}{l}\text { Index patient } \\
\text { with smear } \\
\text { positive disease }\end{array}$ & $\begin{array}{c}\text { Index patient } \\
\text { with smear } \\
\text { negative disease }\end{array}$ \\
\hline \multicolumn{3}{|l|}{6 Months: } \\
\hline \multicolumn{3}{|l|}{ Close contact: } \\
\hline Indian subcontinent & $11 \quad(6)$ & $10(10)$ \\
\hline Other & $15(12)$ & $10 \star(7)$ \\
\hline \multicolumn{3}{|l|}{ Casual contact: } \\
\hline Indian subcontinent & 0 & $8^{\star}(6)$ \\
\hline Other & $13^{\star}(11)$ & $3^{\star}(3)$ \\
\hline \multicolumn{3}{|l|}{12 Months: } \\
\hline \multicolumn{3}{|l|}{ Close contact: } \\
\hline Indian subcontinent & $5 \quad(1)$ & 9 (2) \\
\hline Other & $12 \quad(5)$ & $6^{\star}(2)$ \\
\hline \multicolumn{3}{|l|}{ Casual contact: } \\
\hline Indian subcontinent & 0 & 0 \\
\hline Other & $5^{\star}(5)$ & $3^{\star}(3)$ \\
\hline \multicolumn{3}{|l|}{24 Months: } \\
\hline \multicolumn{3}{|l|}{ Close contact: } \\
\hline Indian subcontinent & $1 \quad(0)$ & \\
\hline & $5^{\star}(3)$ & \\
\hline \multicolumn{3}{|l|}{ Casual contact: } \\
\hline $\begin{array}{l}\text { Indian subcontinent } \\
\text { Other }\end{array}$ & $\begin{array}{ll}0 \\
4^{\star} & (0)\end{array}$ & \\
\hline
\end{tabular}

^Inappropriate follow up appointments. 
Follow up after initial screening should also have been determined on the basis of ethnic origin and closeness of contact. However, we found that ethnic origin was not routinely recorded and contacts were not clearly classified as close or casual (apart from name and address).

\section{BRITISH THORACIC SOCIETY GUIDELINES}

The British Thoracic Society continues to recommend follow up of close contacts (those unvaccinated with Heaf grade 2 and all with Heaf grade 3-4) for up to two years. ${ }^{2}$ In our study all five asymptomatic contacts with tuberculosis had it diagnosed on initial screening, at which time the decision to give chemoprophylaxis to another four contacts was made. In the light of these results and similar low yields from other contact studies $^{3-5}$ prolonged follow up seems to be unnecessary in districts like South Glamorgan with small immigrant populations.

The $1978,{ }^{1} 1983,{ }^{6}$ and $1990^{2}$ British Thoracic Society guidelines recommend screening casual contacts only if the index patient is highly infectious, yet the term is undefined. If "highly infectious index case" were to be defined as an index patient who had at least one close contact with tuberculosis diagnosed then the casua contacts of $94 \%$ of index patients need not have been screened. This would have meant 253 fewer contacts being screened, resulting in 184 fewer Heaf tests and 151 fewer chest radiographs. This would have saved the health authority $£ 6000-£ 8000$ in direct costs alone (Heaf tests and radiographs). Time and effort spent on tracing contacts would have been cut, together with the administrative work of maintaining records, filing results, and sending clinic appointments. Over 300 clinic attendances could have been avoided, thus reducing the contact clinic schedule by around 15 sessions and allowing redeployment of the contact team to other community health service activities.

\section{IMPROVING PERFORMANCE}

We recommend that to improve the efficiency of tuberculosis contact tracing the notification form should specify the smear test result and ethnic origin of the index patient. Classification of contacts into close or casual should be clearly recorded by the contact team. Screening can then be cost effectively focused on those at greatest risk of developing tuberculosis.

In the light of the results of this study changes incorporating the above recommendations have been made in the contact tracing procedure in South Glamorgan. A further retrospective study is planned in two years time to evaluate these changes in practice and thus complete the audit feedback loop.

\footnotetext{
1 British Thoracic and Tuberculosis Association. A study of a standardized contact procedure in tuberculosis. Tubercle 1978;59:245-59.

2 Subcommitree of the Joint Tuberculosis Commitree of the British Thoracic Socity. Contol and prevention of uberculosis Britain: Brish Thoracic Society. Control and prevention of

3 Spencer Jones J. Tuberculosis case finding in coastal south-east Kent, 1977-81. Lancet 1983; ; $232-3$.

4 Selby $\mathrm{CD}$, Allen $\mathrm{MB}$, Leitch AG. Tuberculosis contact tracing in Edinburgh. Thorax 1988;43:834P.

5 Esmonde TFG, Petheram IS. Audit of tuberculosis contact tracing procedure in South Gwent. Respir Med 1991;85:421-4.

6 Joint Tuberculosis Committee. Control and prevention of tuberculosis: a code of practice. BMF 1983;287:1118-21.

(Accepled 24 February 1992)
}

\title{
Must diabetes be a fatal disease in Africa? Study of costs of treatment
}

\author{
Stella S Chale, Andrew B M Swai, Phare G M Mujinja, Donald G McLarty
}

Department of Medicine, Muhimbili Medical Centre, PO Box 65001, Dar es Salaam, Tanzania Stella S Chale, lecturer in medicine

Andrew B M Swai, senior lecturer in medicine Donald G McLarty, professor of medicine

Department of Behavioural Sciences, Muhimbili Medical Centre, Dar es Salaam, Tanzania

Phare G M Mujinja, lecturer in health economics

Correspondence to: Dr Chale.

$B M \mathcal{F}$ 1992;304:1215-8

\section{Abstract}

Objective-To estimate the costs of diagnosis and treatment of diabetes in Tanzania.

Design-Costs estimated from the reported and recorded experience of patients with newly presenting diabetes in 1989-90 and of diabetic patients first seen in 1981-2.

Setting-Muhimbili Medical Centre, Dar es Salaam.

Subjects -464 patients ( 315 men and 149 women). 262 patients diagnosed during 1 September 1989-31 August 1990 (group 1) and 202 during 1 June 1981-31 August 1982 (group 2).

Results - The average annual direct cost of diabetes care in $1989-90$ was $\$ 287$ for a patient requiring insulin and $\$ 103$ for a patient not requiring insulin. Purchase of insulin accounted for $68.2 \%(\$ 156)$ of the average annual outpatient costs for patients requiring insulin. For patients not requiring insulin the cost of oral hypoglycaemic drugs and treatment of chronic complications and infections accounted for $42.5 \%(\$ 29.3)$ and $48.8 \%(\$ 33.7)$ of costs respectively. Cost of outpatient care of diabetic patients for the whole of Tanzania was estimated at $\$ 2.7 \mathrm{~m}, \$ 875128$ $(32.2 \%)$ of which was for insulin. Doctors' and nurses' costs accounted for $\mathbf{0 . 2} \%$ of total costs of outpatient care. The annual direct inpatient care costs were estimated at $\$ 1 \cdot 25 \mathrm{~m}$. Around $0.2 \%$ of the Tanzanian population aged 15 years and over used the equivalent of $8 \%$ of the total government health expenditure, which was $\$ 47408382$.

Conclusion-Diabetes places a severe strain on the limited resources of developing countries. If
African patients with diabetes have to pay for their treatment most will be unable to do so and will die.

\section{Introduction}

People with diabetes mellitus are known to be heavy lifetime users of health care resources, ${ }^{1-4}$ but few studies have considered the economic implications of this for developing countries. ${ }^{15}$ Tanzania (population 23174336 ), with a gross per capita income of US $\$ 160$ to $\$ 200$ per year, is one of the world's 25 poorest countries. Health expenditure per head has fallen from $\$ 7$ in 1980 to $\$ 2$ in 1989 .

Rates of non-infectious diseases such as diabetes in rural Tanzania are among the lowest in the world, ${ }^{78}$ but chronic, non-infectious diseases consume a high proportion of the total health expenditure. The present study was undertaken to estimate the direct and indirect costs of diagnosis, treatment, complications, and follow up of diabetic patients in Dar es Salaam, Tanzania, and from these to estimate the costs of diabetes nationally.

\section{Subjects and methods}

Dar es Salaam is Tanzania's largest city with a population of 1.5 million. Most African patients with diabetes from the city are referred to Muhimbili Medical Centre, the country's largest hospital. Patients who need inpatient care are admitted to the general medical wards, where they are seen and instructed by nurses with special training in diabetes.

Special diabetic outpatient clinics are held every 